\title{
The tandem radical route to indole alkaloids: an unusual rearrangement reaction
}

\author{
Stephen T. Hilton ${ }^{\dagger}$ and Keith Jones ${ }^{* \dagger+}$ \\ School of Chemical and Pharmaceutical Sciences, Kingston University, Penrhyn Road, \\ Kingston-upon-Thames, Surrey, KT1 2EE, UK \\ E-mail: Keith.jones@icr.ac.uk
}

\begin{abstract}
Cyclisation of the precursor 6 under standard radical conditions yields the tetracyclic structure 7 which represents the core of a number of indole alkaloids along with the novel tetracycle $\mathbf{1 3 .}$
\end{abstract}

Keywords: Indoles, radical cyclisation, rearrangement, alkaloids

\section{Introduction}

The Strychnos and Aspidosperma alkaloids are structurally complex molecules that possess a range of biological activity and present a challenge to synthetic organic chemists. ${ }^{1}$ As part of our ongoing interest in these classes of alkaloids, ${ }^{2}$ we are particularly concerned with a synthesis of the core tetracyclic sub-structure 1, which forms the basis of these molecules as exemplified by strychnine $\mathbf{2}$ and aspidospermidine $\mathbf{3}$ (Figure 1).

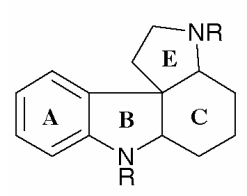

Tetracyclic Core 1

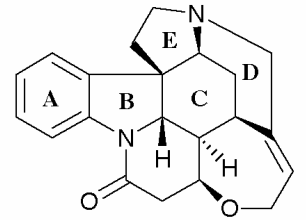

Strychnine 2

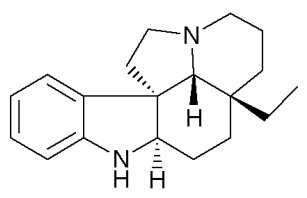

Aspidospermidine 3

\section{Figure 1}

\footnotetext{
${ }^{\dagger}$ Current address: Cancer Research UK Centre for Cancer Therapeutics, The Institute of Cancer Research, 15 Cotswold Road, Sutton, Surrey, SM2 5NG. UK

${ }^{*}$ Keith Jones is Chairman (2005-2007) of the RSC Heterocyclic Group and was Secretary and Treasurer during the period 1996-1999.'
} 
In our previous studies, we had shown that tandem radical cyclisation of the precursor 4 gave the tetracyclic structure 5 as a mixture of 4 diastereoisomers (Scheme 1). ${ }^{3}$

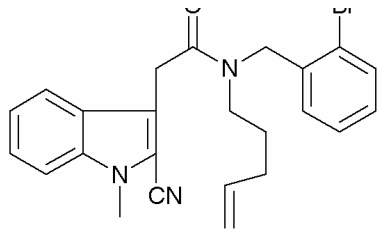

4
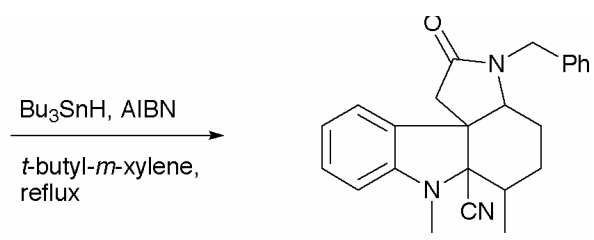

5

\section{Scheme 1}

Whilst this route provided the core tetracyclic structure 5, it possesses limited functionality with which to undertake a synthesis of either the Strychnos or Aspidosperma alkaloids. We therefore embarked on a synthesis of a cyclisation precursor which, after cyclisation, would enable us to access suitable intermediates for the synthesis of these two classes of alkaloids. The proposed cyclisation precursor $\mathbf{6}$ is shown below. It possesses a terminal $\mathrm{C} / \mathrm{C}$ triple bond, which on cyclisation under our standard conditions should provide an exocyclic double bond on the tetracyclic product 7 (Scheme 2). This would allow further elaboration of such molecules towards natural products such as strychnine and aspidospermidine.

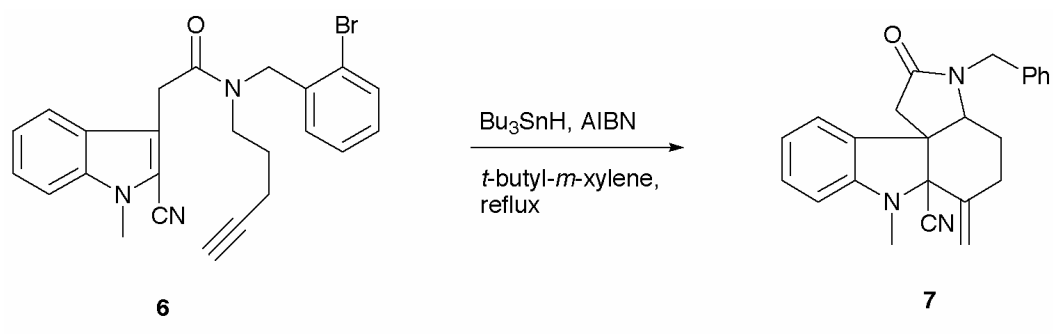

\section{Scheme 2}

\section{Results and Discussion}

The required cyclisation precursor was synthesized following our previous approach from the sulfonamide $\mathbf{8}$ which utilized the work of Bowman and Fukuyama for the synthesis of the secondary amine. ${ }^{4,5}$ Reaction of $\mathbf{8}$ with 4-pentynol under Mitsunobu conditions gave the tertiary sulfonamide 9 in $96 \%$ yield (Scheme 3). Deprotection of the tertiary sulfonamide 9 using potassium carbonate and thiophenol gave the secondary amine $\mathbf{1 0}$ in $75 \%$ yield. ${ }^{5}$ Reaction of this with the acid chloride $\mathbf{1 1}$ following our previous work on oxindoles, ${ }^{6}$ gave the cyclisation precursor $\mathbf{6}$ in $86 \%$ yield as a 1:1.3 mixture of amide rotamers (Scheme 3). 


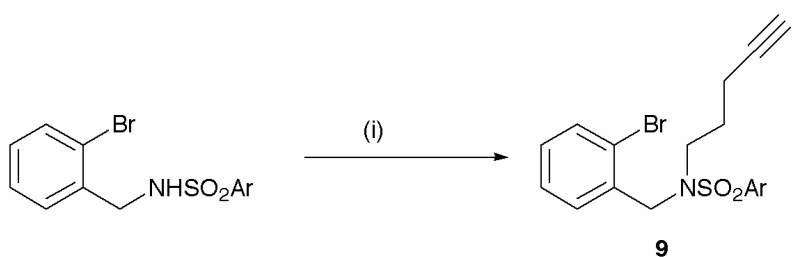

$8(\mathrm{Ar}=2$-nitrophenyl)
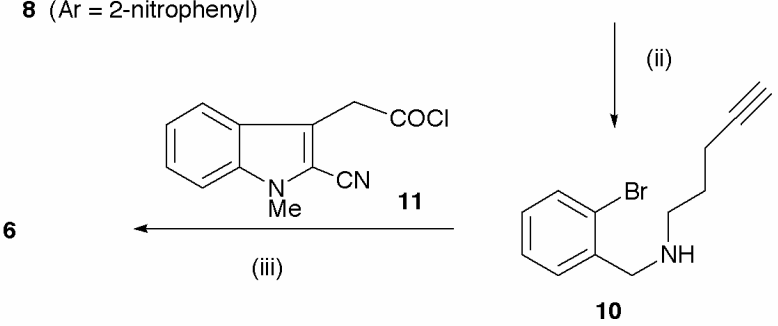

Scheme 3. Reagents and Conditions: (i) $\mathrm{PPh}_{3}$, DEAD, THF, $0{ }^{\circ} \mathrm{C}, 96 \%$; (ii) $\mathrm{K}_{2} \mathrm{CO}_{3}, \mathrm{MeCN}$, $\mathrm{PhSH}, 75 \%$; (iii) $\mathrm{iPr}_{2} \mathrm{NEt}, \mathrm{CH}_{2} \mathrm{Cl}_{2}, 0^{\circ} \mathrm{C}, 86 \%$.

Following the successful cyclisation of the $N$-pentenyl precursor 4 in our previous example, ${ }^{3}$ we decided to use the same conditions for cyclisation of the $N$-pentynyl precursor $\mathbf{6}$. Treatment of a solution of the tertiary amide $\mathbf{6}$ in $t$-butyl- $m$-xylene under reflux with syringe pump addition of a solution of tri- $n$-butyltin hydride and AIBN in $t$-butyl- $m$-xylene resulted in the formation of three isolable products. High-resolution mass spectrometry confirmed the same molecular formula for all three compounds, $\mathrm{C}_{24} \mathrm{H}_{23} \mathrm{~N}_{3} \mathrm{O}$. The least polar compound was identified as the reduced product $\mathbf{1 2}$ owing to the presence of 9 aromatic protons, the terminal acetylene proton and an equal mixture of amide rotamers in the ${ }^{1} \mathrm{H}$ NMR spectrum. It was isolated in $45 \%$ yield. From the ${ }^{13} \mathrm{C}$ NMR spectrum, the second product isolated in $16 \%$ yield clearly possessed an exocyclic double bond (resonance at $\delta$ 119.4) along with the distinctive spirocyclic center $(\delta$ 53.8), indicating that the precursor 6 had undergone the desired translocation-cyclisation-cyclisation sequence onto the exocyclic triple bond. This along with the other observable data such as two distinct $\mathrm{AB}$ quartets in the ${ }^{1} \mathrm{H}$ NMR clearly indicated that we had indeed synthesized the tetracyclic structure 7. The relative stereochemistry of this diastereoisomer cannot easily be determined owing to the three contiguous quaternary centers but it appears to be one single diastereoisomer.

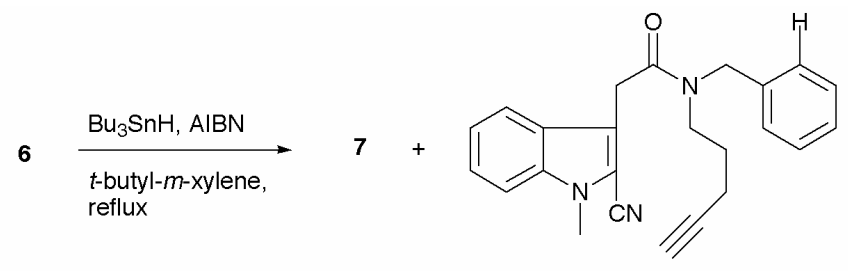

12

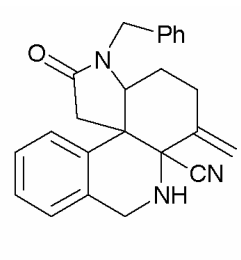

13

\section{Scheme 4}


The nature of the third isolable product presented a challenge, as it clearly possessed three AB quartets from the ${ }^{1} \mathrm{H}$ NMR spectrum. The exocyclic double bond was also present indicating that the final cyclisation step had taken place. From the ${ }^{13} \mathrm{C}$ NMR spectrum there were $5 \mathrm{CH}_{2}$ signals excluding the exocyclic methylene group. Eight quaternary carbons were also observed in the ${ }^{13} \mathrm{C}$ NMR spectrum with no evidence for the expected $N$-methyl group in either the ${ }^{1} \mathrm{H}$ or ${ }^{13} \mathrm{C}$ NMR spectrum. On the basis of the evidence, we have assigned the tetracyclic structure 13 to this compound, which was isolated in $15 \%$ yield. The formation of this unexpected product is believed to have occurred by an additional 1,5-hydrogen atom abstraction by the vinylic radical 17 from the neighbouring $N$-methyl group of the indole (Scheme 5). The resultant primary radical 18 then undergoes a neophyl-type rearrangement by addition to the benzene ring to give cyclohexadienyl radical 19. Ring-opening of $\mathbf{1 9}$ to regenerate the aromatic ring gives an aminyl radical which is reduced by tri- $n$-butyltin hydride to give the final compound $\mathbf{1 3}$.<smiles>C#CCCCN(Cc1ccccc1)C(=O)Cc1c(C#N)n(C)c2ccccc12</smiles>
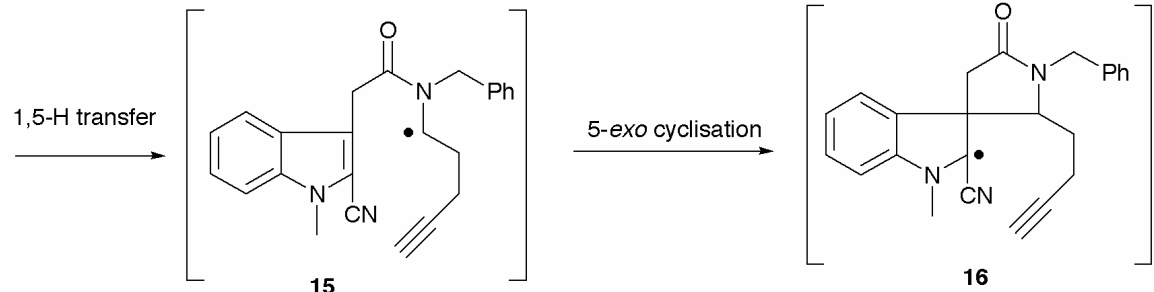

6-exo cyclisation

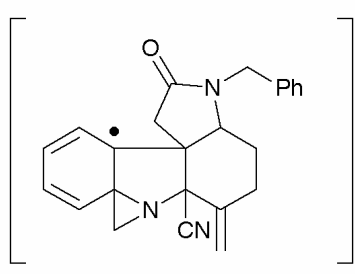

19<smiles>C=C1CCC2N(Cc3ccccc3)C(=O)CC23c2ccccc2CN(C)C13C</smiles>

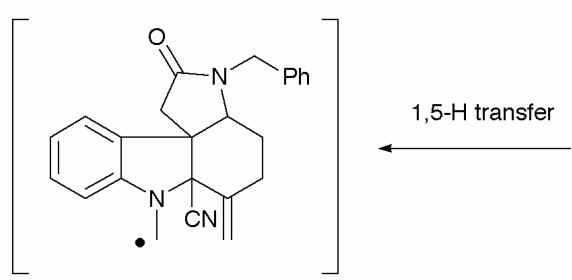

18

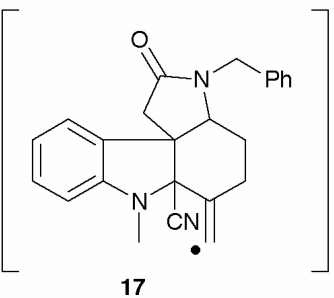

17

\section{Scheme 5}


This unexpected product $\mathbf{1 3}$ possesses a novel ring structure which has arisen by a formal ring expansion of an indole to a tetrahydroisoquinoline. The formation of this product is surprising. 1,5-Hydrogen atom abstraction by a vinylic radical is well precedented ${ }^{7-9}$ as is the neophyl rearrangement involving an aromatic ring with concomitant ring expansion. ${ }^{10,11}$ However, the sequential occurrence of these two reactions as part of a tandem process is unprecedented.

In summary, cyclisation of the $N$-pentynyl precursor 6 results in the formation of the expected cyclised product 7 , carrying suitable functionality for conversion into natural indole alkaloids. In addition, the novel tetracycle $\mathbf{1 3}$ has also been isolated. We suggest that this arises through a combination of hydrogen atom abstraction and radical rearrangement.

\section{Experimental Section}

General Procedures. ${ }^{1} \mathrm{H}$ NMR and ${ }^{13} \mathrm{C}$ NMR were recorded on a Bruker AM300 spectrometer operating at $300 \mathrm{MHz}$ for proton and $75 \mathrm{MHz}$ for carbon. Infrared spectra were recorded on a Perkin Elmer 1605 FT-IR spectrophotometer using $\mathrm{NaCl}$ plates. High resolution mass spectra were carried out at King's College, London University or at Kingston University. Mass spectra carried out at King's College using either a Kratos MS89MS with Kratos DS90 software or a Jeol AX505W with Jeol complement data system. Samples were ionised electronically (EI), with an accelerating voltage of $\approx 6 \mathrm{kV}$ or by low resolution fast atom bombardment (FAB) in a thioglycerol matrix. High resolution fast atom bombardment was carried out at the ULIRS mass spectrometry facility at the School of Pharmacy, University of London. Mass spectra carried out at Kingston University were recorded using a Micromass LCT time of flight mass spectrometer equipped with an ElectroSpray Ionisation (ESI) ion source.

N-(2-Bromobenzyl)-2-nitrobenzenesulfonamide (8). A solution of 2-nitrobenzene sulfonyl chloride $(25.00 \mathrm{~g}, 112.3 \mathrm{mmol})$ in dichloromethane $\left(100 \mathrm{~cm}^{3}\right)$ was added dropwise to a stirred solution of 2-bromobenzylamine hydrochloride $(25.00 \mathrm{~g}, 112.3 \mathrm{mmol})$ and triethylamine $(50.1$ $\left.\mathrm{cm}^{3}, 36.40 \mathrm{~g}, 360.0 \mathrm{mmol}\right)$ in dichloromethane $\left(150 \mathrm{~cm}^{3}\right)$ at $0{ }^{\circ} \mathrm{C}$. The resulting solution was allowed to warm to room temperature and stirred for 16 hours, washed with water $\left(3 \mathrm{x} 200 \mathrm{~cm}^{3}\right)$, dried $\left(\mathrm{MgSO}_{4}\right)$, filtered and organic solvent removed under reduced pressure. The crude product was purified by recrystallisation (ethyl acetate/ hexane) to give $N$-(2-bromobenzyl)-2nitrobenzenesulfonamide (8) as a white crystalline solid (36.63 g, 89\%); m.p. 101-103 ${ }^{\circ} \mathrm{C}$; $\mathrm{R}_{\mathrm{f}}(3$ : 2, hexane: ethyl acetate) 0.57; (Found $\mathrm{MH}^{+}, 370.9694 / 372.9696, \mathrm{C}_{13} \mathrm{H}_{12} \mathrm{BrN}_{2} \mathrm{O}_{4} \mathrm{~S}$ requires $370.9701 / 372.9681) ; v_{\max } / \mathrm{cm}^{-1} 3347.8(\mathrm{NH}), 1531.1\left(\mathrm{Ar}-\mathrm{NO}_{2}\right), 1162.6\left(\mathrm{NSO}_{2} \mathrm{Ar}\right) ; \delta_{\mathrm{H}}(300$ $\left.\mathrm{MHz} ; \mathrm{CDCl}_{3}\right) 4.34\left(2 \mathrm{H}, \mathrm{d}, J 6.3, \mathrm{CH}_{2}\right), 6.04(1 \mathrm{H}, \mathrm{t}, J 6.3, \mathrm{NH}), 7.00(1 \mathrm{H}, \mathrm{td}, J 7.8$ \& 1.6, C-4H), $7.13(1 \mathrm{H}, \mathrm{td}, J 7.8 \& 1.6, \mathrm{C}-5 H), 7.29(1 \mathrm{H}, \mathrm{dd}, J 7.8 \& 1.6, \mathrm{C}-6 H), 7.31(1 \mathrm{H}, \mathrm{dd}, J 7.8 \& 1.6, \mathrm{C}-$ $3 H), 7.51\left(1 \mathrm{H}, \mathrm{td}, J 7.6 \& 1.6, \mathrm{C}-5^{\prime} H\right), 7.58\left(1 \mathrm{H}, \mathrm{td}, J 7.6 \& 1.6, \mathrm{C}-4^{\prime} H\right), 7.34(1 \mathrm{H}, \mathrm{dd}, J 7.6 \&$ 1.6, C-6'H), $7.83\left(1 \mathrm{H}, \mathrm{dd}, J 7.6 \& 1.6, \mathrm{C}-3^{\prime} H\right) ; \delta_{\mathrm{C}}\left(75 \mathrm{MHz} ; \mathrm{CDCl}_{3}\right) 48.20\left(\mathbf{C H}_{2}\right), 123.60(\mathbf{C}-2)$, 
125.40 (Ar-C-H), 127.63 (Ar-C-H), 129.80 (Ar-C-H), 130.72 (Ar-C-H), 130.92 (Ar-C-H), 132.80 (Ar-C-H), 132.91 (Ar-C-H), 133.61 (Ar-C-H), 133.86 (quaternary C), 134.93 (quaternary C), 147.48 (C-2').

$\mathrm{N}$-(2-Bromobenzyl)-2-nitro- $\mathrm{N}$-pent-4-ynylbenzenesulfonamide (9). Diethyl azodicarboxylate $\left(11.20 \mathrm{~cm}^{3}, 12.38 \mathrm{~g}, 71.1 \mathrm{mmol}\right)$ was added dropwise to a solution of $N$-(2-bromobenzyl)-2nitrobenzenesulfonamide (8) $(20.30 \mathrm{~g}, 54.7 \mathrm{mmol})$, 4-pentyn-1-ol $\left(6.1 \mathrm{~cm}^{3}, 5.52 \mathrm{~g}, 65.6 \mathrm{mmol}\right)$ and triphenylphosphine $(18.64 \mathrm{~g}, 71.1 \mathrm{mmol})$ in THF $\left(150 \mathrm{~cm}^{3}\right)$ at $0{ }^{\circ} \mathrm{C}$. The reaction was allowed to warm to room temperature and stirred overnight. Organic solvent was removed under reduced pressure and the residue taken up in diethyl ether $\left(70 \mathrm{~cm}^{3}\right)$. The volume of the solution was reduced and the solution filtered to remove triphenylphosphine oxide. The filtrate was reduced in volume and the yellow oil purified by flash column chromatography (3:2, hexane: ethyl acetate) to give the title compound (9) as a light yellow oil $(22.96 \mathrm{~g}, 96 \%) ; \mathrm{R}_{\mathrm{f}}(3: 2$, hexane: ethyl acetate) 0.55; (Found $\mathrm{MH}^{+}, 437.0156 / 439.0169, \mathrm{C}_{18} \mathrm{H}_{18} \mathrm{BrN}_{2} \mathrm{O}_{4} \mathrm{~S}$ requires 437.0171/ 439.0150); $v_{\max } / \mathrm{cm}^{-1} 3300.4(\mathrm{CCH}), 3061.0,2942.5(\mathrm{Ar}-\mathrm{H}), 1544.7\left(\mathrm{Ar}^{\left.-\mathrm{NO}_{2}\right), 1371.4}\right.$ $\left(\mathrm{NSO}_{2} \mathrm{Ar}\right), 1163.8\left(\mathrm{NSO}_{2} \mathrm{Ar}\right) ; \delta_{\mathrm{H}}\left(300 \mathrm{MHz} ; \mathrm{CDCl}_{3}\right) 1.61\left(2 \mathrm{H}, \mathrm{m}, \mathrm{NCH}_{2} \mathrm{CH}_{2}\right), 1.87(1 \mathrm{H}, \mathrm{t}, J 2.6$, $\mathrm{CCH}), 2.04\left(2 \mathrm{H}, \mathrm{td}, J 7.0 \& 2.6, \mathrm{CH}_{2} \mathrm{CH}_{2} \mathrm{CCH}\right), 3.39\left(2 \mathrm{H}, \mathrm{t}, J 7.6, \mathrm{NCH}_{2} \mathrm{CH}_{2}\right), 4.66(2 \mathrm{H}, \mathrm{s}$, $\left.\mathrm{NCH}_{2} \mathrm{Ar}\right), 7.09(1 \mathrm{H}, \mathrm{td}, J 7.6 \& 1.6, \mathrm{C}-4 H), 7.23(1 \mathrm{H}, \mathrm{td}, J 7.6 \& 1.6, \mathrm{C}-5 H), 7.42(1 \mathrm{H}, \mathrm{dd}, J 7.6$ \& 1.6, C-6H), $7.47(1 \mathrm{H}, \mathrm{dd}, J 7.6$ \& 1.6, C-3H), 7.60-7.70 $(3 \mathrm{H}, \mathrm{m}, \mathrm{Ar}-H), 7.96(1 \mathrm{H}, \mathrm{m}, \mathrm{Ar}-H)$; $\delta_{\mathrm{C}}\left(75 \mathrm{MHz} ; \mathrm{CDCl}_{3}\right) 15.73\left(\mathrm{CH}_{2}\right), 26.78\left(\mathrm{CH}_{2}\right), 47.28\left(\mathrm{NCH}_{2} \mathrm{CH}_{2}\right), 51.48\left(\mathrm{NCH}_{2} \mathrm{Ar}\right), 69.44$ $(\mathbf{C C H}), 82.69(\mathrm{CCH}), 123.32(\mathbf{C}-2), 124.33(\mathrm{Ar}-\mathbf{C}-\mathrm{H}), 127.91$ (Ar-C-H), 129.47 (Ar-C-H), 129.80 (Ar-C-H), 130.86 (Ar-C-H), 132.04 (Ar-C-H), 132.96 (Ar-C-H), 133.09 (C-1), 133.96 (Ar-C-H), 135.02 (C-1'), 147.88 (C-2').

$N$-(2-Bromobenzyl)pent-4-ynylamine (10). Thiophenol $\left(5.9 \mathrm{~cm}^{3}, 6.36 \mathrm{~g}, 57.8 \mathrm{mmol}\right)$ was added to a stirred solution of sulfonamide (9) $(22.95 \mathrm{~g}, 52.5 \mathrm{mmol})$ and potassium carbonate $(23.58 \mathrm{~g}, 170.6 \mathrm{mmol})$ in acetonitrile $\left(150 \mathrm{~cm}^{3}\right)$ at room temperature. The resulting solution was left to stir overnight at room temperature. Organic solvent was removed under reduced pressure and the residue was taken up in diethyl ether $\left(200 \mathrm{~cm}^{3}\right)$. Hydrochloric acid $(2.0 \mathrm{M}$ solution) was added until the solution remained acidic and the resulting solution stirred for ten minutes. The organic layer was separated and washed with dilute hydrochloric acid $\left(100 \mathrm{~cm}^{3}\right.$ of a $1.0 \mathrm{M}$ solution). The combined aqueous phases were washed with diethyl ether $\left(200 \mathrm{~cm}^{3}\right)$ and made basic by the addition of solid potassium carbonate. The aqueous solution was extracted with diethyl ether $\left(4 \times 150 \mathrm{~cm}^{3}\right)$ and the combined extracts washed with brine $\left(200 \mathrm{~cm}^{3}\right)$, dried $\left(\mathrm{MgSO}_{4}\right)$, filtered and evaporated to dryness under reduced pressure to give the title compound (10) as a pale yellow oil $(9.94 \mathrm{~g}, 75 \%) ; \mathrm{R}_{\mathrm{f}}\left(3: 2\right.$, hexane: ethyl acetate) 0.21; (Found $\mathrm{MH}^{+}$, 252.0377/ 254.0381, $\mathrm{C}_{12} \mathrm{H}_{15} \mathrm{BrN}$ requires 252.0388/254.0367); $v_{\max } / \mathrm{cm}^{-1} 3422.5(\mathrm{NH}), 3301.6$ $(\mathrm{CCH}) ; \delta_{\mathrm{H}}\left(300 \mathrm{MHz} ; \mathrm{CDCl}_{3}\right) 1.39(1 \mathrm{H}$, br s, $\left.\mathrm{NH}), 1.64(2 \mathrm{H} \text {, quin, } J 7.0, \mathrm{NHCH} 2 \mathrm{CH})_{2}\right), 1.86(1 \mathrm{H}$, t, $J$ 2.6, $\mathrm{CCH}), 2.19\left(2 \mathrm{H}, \mathrm{td}, J 7.0 \& 2.6, \mathrm{CH}_{2} \mathrm{CCH}\right), 2.64\left(2 \mathrm{H}, \mathrm{t}, J 7.0, \mathrm{NCH}_{2} \mathrm{CH}_{2}\right), 3.76(2 \mathrm{H}, \mathrm{s}$, $\left.\mathrm{NCH}_{2} \mathrm{Ar}\right), 7.01(1 \mathrm{H}, \mathrm{td}, J 7.5 \& 1.0, \mathrm{C}-4 H), 7.17(1 \mathrm{H}, \mathrm{td}, J 7.5 \& 1.0, \mathrm{C}-5 H), 7.29(1 \mathrm{H}, \mathrm{dd}, J 7.5$ \& 1.0, C-6H), $7.43(1 \mathrm{H}, \mathrm{dd}, J 7.5 \& 1.0, \mathrm{C}-3 H) ; \delta_{\mathrm{C}}\left(75 \mathrm{MHz} ; \mathrm{CDCl}_{3}\right) 16.61\left(\mathrm{CH}_{2}\right), 28.75\left(\mathrm{CH}_{2}\right)$, 
$47.92\left(\mathrm{NCH}_{2} \mathrm{CH}_{2}\right), 53.65\left(\mathrm{NCH}_{2} \mathrm{Ar}\right), 68.61(\mathbf{C C H}), 84.08(\mathrm{CCH}), 123.94(\mathbf{C}-2), 127.39(\mathbf{C}-5)$, 128.52 (C-4), 130.19 (C-6), 132.75 (C-3), 139.34 (C-1).

$\mathrm{N}$-(2-Bromobenzyl)-2-(2-cyano-1-methyl-1H-indol-3-yl)- $\mathrm{N}$-pent-4-ynylacetamide (6). A solution of acid chloride (11) $(3.25 \mathrm{~g}, 14.0 \mathrm{mmol})$ in dichloromethane $\left(50 \mathrm{~cm}^{3}\right)$ was added dropwise via cannula to a stirred solution of (2-bromobenzyl)-pent-4-ynylamine (10) (4.58 g, $18.15 \mathrm{mmol})$ and $N, N$-diisopropylethylamine $\left(8.5 \mathrm{~cm}^{3}, 6.31 \mathrm{~g}, 48.9 \mathrm{mmol}\right)$ in dichloromethane $\left(100 \mathrm{~cm}^{3}\right)$ at $0{ }^{\circ} \mathrm{C}$ under nitrogen. The resulting mixture was left to stir at $0{ }^{\circ} \mathrm{C}$ for one hour, allowed to warm to room temperature and then left to stir overnight. Organic solvent was removed under reduced pressure and the residue taken up in dichloromethane $\left(200 \mathrm{~cm}^{3}\right)$, washed with $\mathrm{HCl}\left(200 \mathrm{~cm}^{3}\right.$ of a $0.5 \mathrm{M}$ solution), saturated sodium hydrogen carbonate $\left(200 \mathrm{~cm}^{3}\right)$ and brine $\left(200 \mathrm{~cm}^{3}\right)$. The organic phase was dried $\left(\mathrm{MgSO}_{4}\right)$, filtered and solvent removed under reduced pressure to give a light green solid which was purified by flash column chromatography (2: 1, hexane: ethyl acetate) to give the tertiary amide (6) as a light yellow solid and as a 1: 1.3 ratio of amide rotamers $(5.40 \mathrm{~g}, 86 \%)$; m.p. $119-120{ }^{\circ} \mathrm{C} ; \mathrm{R}_{\mathrm{f}}(2: 1$, hexane: ethyl acetate) 0.83 ; (Found $\mathrm{M}^{+}$, 447.0956/449.0916, $\mathrm{C}_{24} \mathrm{H}_{22} \mathrm{BrN}_{3} \mathrm{O}$ requires 447.0947/449.0927); $v_{\max } / \mathrm{cm}^{-1} 3303.1$ $(\mathrm{CCH}), 3012.8(\mathrm{Ar}-\mathrm{H}), 2217.6(\mathrm{CN}), 1653.9\left(\mathrm{CONR}_{2}\right) ; \delta_{\mathrm{H}}\left(300 \mathrm{MHz} ; \mathrm{CDCl}_{3}\right) 1.96(2 \mathrm{H}$, quin, $J$ 6.8, C-2' ' $\mathrm{H}_{2}$ (major + minor)), $2.08(0.4 \mathrm{H}, \mathrm{t}, J 2.4, \mathrm{C}-5$ ' ' $\mathrm{H}$ (minor)), 2.17 (0.6H, t, J 2.4, C-5' 'H (major)), 2.34 (1.2H, td, $J 6.8 \& 2.4, \mathrm{C}-3$ ' ' $\mathrm{H}_{2}$ (major)), 2.40 (0.8H, td, $J 6.8 \& 2.4, \mathrm{C}-3$ ' ' $\mathrm{H}_{2}$ (minor)), 3.64 (2H, m, C-1' ' $\mathrm{H}_{2}$ (major + minor)), $3.83\left(1.8 \mathrm{H}, \mathrm{s}, \mathrm{NCH}_{3}\right.$ (major)), 3.97 (1.2H, s, $\mathrm{NCH}_{3}$ (minor)), $4.02\left(1.2 \mathrm{H}, \mathrm{s}, \mathrm{CH}_{2} \mathrm{CO}\right.$ (major)), $4.25\left(0.8 \mathrm{H}, \mathrm{s}, \mathrm{CH} \mathrm{C}_{2} \mathrm{CO}\right.$ (minor)), $4.82(1.2 \mathrm{H}, \mathrm{s}$, $\mathrm{NCH}_{2} \mathrm{Ar}$ (major)), $4.88\left(0.8 \mathrm{H}, \mathrm{s}, \mathrm{NCH}_{2} \mathrm{Ar}\right.$ (minor)), $6.92(0.4 \mathrm{H}, \mathrm{m}, \mathrm{Ar}-\mathrm{H}$ (minor)), 7.04-7.42 $(5.6 \mathrm{H}, \mathrm{m}, \mathrm{Ar}-H$ (major + minor)), $7.49(0.4 \mathrm{H}, \mathrm{d}, J$ 8.0, Ar- $H$ (minor)), $7.70(0.6 \mathrm{H}, \mathrm{m}, \mathrm{Ar}-H$ (major)), $7.76\left(0.6 \mathrm{H}, \mathrm{d}, J\right.$ 8.0, Ar- $H$ (major)), $7.89\left(0.4 \mathrm{H}, \mathrm{d}, J\right.$ 8.0, Ar- $H$ (minor)); $\delta_{\mathrm{C}}(75 \mathrm{MHz}$; $\left.\mathrm{CDCl}_{3}\right) 15.86\left(\mathrm{CH}_{2}\right), 16.17\left(\mathrm{CH}_{2}\right), 26.33\left(\mathrm{CH}_{2}\right), 27.36\left(\mathrm{CH}_{2}\right), 30.76\left(\mathrm{CH}_{2}\right), 31.09\left(\mathrm{CH}_{2}\right), 31.41$ $\left(\mathrm{NCH}_{3}\right), 31.62\left(\mathrm{NCH}_{3}\right), 46.50\left(\mathrm{CH}_{2}\right), 46.78\left(\mathrm{CH}_{2}\right), 48.65\left(\mathrm{CH}_{2}\right), 52.57\left(\mathbf{C H}_{2}\right), 69.12\left(\mathbf{C}-4{ }^{\prime \prime}\right)$, 70.00 (C-4'), 82.72 (C-5'), 83.40 (C-5'), 109.82 (C-3), 110.06 (C-7), 110.14 (C-7), 112.93 $(\mathbf{C N}), 113.32(\mathbf{C N}), 120.09$ (quaternary C), 120.69 (quaternary C), 121.08 (Ar-C-H), 121.18 (Ar-C-H), 121.43 (Ar-C-H), 122.60 (quaternary C), 123.50 (quaternary C), $126.07+126.22+$ $126.77+127.66+127.33+128.85+128.99+129.21+132.75+133.08($ Ar-C-H), 135.33 (quaternary C), 136.25 (quaternary C), 137.96 (quaternary C), 138.14 (quaternary $\mathbf{C}$ ), 169.43 (CO), 169.84 (CO); $m / z 449.09$ (18.8\%, $\left.\mathrm{M}^{+81} \mathrm{Br}\right), 447.10$ (20.6, $\left.\mathrm{M}^{+79} \mathrm{Br}\right), 368.17$ (62.9), 183.09 (59.2), 170.99 (91.8), 170.10 (66.6), 169.09 (100.0), 168.08 (43.3), 91.06 (49.9), 90.05 (56.0).

Radical cyclisation of $\boldsymbol{N}$-(2-bromobenzyl)-2-(2-cyano-1-methyl-1H-indol-3-yl)- $\boldsymbol{N}$-pent-4ynylacetamide (6). A solution of tri- $n$-butyltin hydride $\left(0.75 \mathrm{~cm}^{3}, 0.81 \mathrm{~g}, 2.78 \mathrm{mmol}\right)$ and azobis-isobutyronitrile (AIBN) $(0.048 \mathrm{~g}, 2.78 \mathrm{mmol})$ in $t$-butyl- $m$-xylene $\left(30 \mathrm{~cm}^{3}\right)$ were added dropwise via syringe pump $\left(6.37 \mathrm{~cm}^{3} /\right.$ hour $)$ to a solution of the amide (6) (1.039 $\left.\mathrm{g}, 2.32 \mathrm{mmol}\right)$ in $t$-butyl- $m$-xylene $\left(90 \mathrm{~cm}^{3}\right)$ at reflux. Once addition was complete, heating was continued for 2 hours. The reaction was allowed to cool and organic solvent removed under reduced pressure and the residue taken up in acetonitrile $\left(50 \mathrm{~cm}^{3}\right)$ and extracted with hexane $\left(4 \times 50 \mathrm{~cm}^{3}\right)$. The 
acetonitrile was removed under reduced pressure and the residue purified by multiple flash column chromatography (3: 1 , hexane: ethyl acetate) to give a mixture of three products:

$\mathbf{N}$-benzyl-2-(2-cyano-1-methyl-1H-indol-3-yl)-N-(pent-4-ynyl)acetamide (12). White solid as a 1: 1 mix of amide rotamers (0.38 g, 45\%); m.p. 82-84 ${ }^{\circ} \mathrm{C}$; (Found $\mathrm{M}^{+}, 369.18510, \mathrm{C}_{24} \mathrm{H}_{23} \mathrm{~N}_{3} \mathrm{O}$ requires 369.18411); $v_{\max } / \mathrm{cm}^{-1} 3296.7(\mathrm{CCH}), 3059.2,3030.5,2938.7(\mathrm{Ar}-\mathrm{H}), 2217.5(\mathrm{CN})$, $1648.6\left(\mathrm{CONR}_{2}\right) ; \delta_{\mathrm{H}}\left(300 \mathrm{MHz} ; \mathrm{CDCl}_{3}\right) 1.81\left(2 \mathrm{H}\right.$, quin, $J$ 7.0, C-2' $\left.{ }^{\prime} \mathrm{H}_{2}\right), 1.94(0.5 \mathrm{H}, \mathrm{t}, J$ 2.5, $\mathrm{CCH}), 2.05(0.5 \mathrm{H}, \mathrm{t}, J 2.5, \mathrm{CCH}), 2.19\left(1 \mathrm{H}, \mathrm{td}, J 7.0 \& 2.5, \mathrm{C}-3{ }^{\prime}{ }^{\prime} \mathrm{H}_{2}\right), 2.26(1 \mathrm{H}, \mathrm{td}, J 7.0 \& 2.5$, C-3' ' $\left.\mathrm{H}_{2}\right), 3.51\left(2 \mathrm{H}, \mathrm{m}, \mathrm{C}-1{ }^{\prime}{ }^{\prime} \mathrm{H}_{2}\right), 3.72(1.5 \mathrm{H}, \mathrm{s}, \mathrm{NCH}), 3.85\left(1.5 \mathrm{H}, \mathrm{s}, \mathrm{NCH}_{3}\right), 3.96(1 \mathrm{H}, \mathrm{s}$, $\left.\mathrm{CH}_{2} \mathrm{CO}\right), 4.09\left(1 \mathrm{H}, \mathrm{s}, \mathrm{CH}_{2} \mathrm{CO}\right), 4.65\left(1 \mathrm{H}, \mathrm{s}, \mathrm{CONCH}_{2} \mathrm{Ar}\right), 4.72\left(1 \mathrm{H}, \mathrm{s}, \mathrm{CONCH}_{2} \mathrm{Ar}\right), 7.09-7.44$ $(8 \mathrm{H}, \mathrm{m}, \mathrm{Ar}-H), 7.69(0.5 \mathrm{H}, \mathrm{d}, J$ 8.1, Ar- $H), 7.77(0.5 \mathrm{H}, \mathrm{d}, J 8.1, \mathrm{Ar}-H) ; \delta_{\mathrm{C}}\left(75 \mathrm{MHz} ; \mathrm{CDCl}_{3}\right)$ $15.80\left(\mathrm{CH}_{2}\right), 16.14\left(\mathrm{CH}_{2}\right), 26.28\left(\mathrm{CH}_{2}\right), 27.15\left(\mathrm{CH}_{2}\right), 30.77\left(\mathbf{C H}_{2}\right), 31.27\left(\mathbf{C H}_{2}\right), 31.44\left(\mathrm{NCH}_{3}\right)$, $31.62\left(\mathrm{NCH}_{3}\right), 46.15\left(\mathrm{CH}_{2}\right), 46.37\left(\mathrm{CH}_{2}\right), 48.72\left(\mathrm{CH}_{2}\right), 51.91\left(\mathrm{CH}_{2}\right), 69.12(\mathbf{C C H}), 69.94$ $(\mathbf{C C H}), 82.84(\mathrm{CCH}), 83.54(\mathrm{CCH}), 109.76(\mathbf{C}-3), 110.05(\mathbf{C}-7), 110.15(\mathbf{C}-7), 113.21(\mathbf{C N})$, $113.38(\mathbf{C N}), 120.48$ (quaternary C), 120.91 (quaternary C), 121.20 (Ar-C-H), 121.29 (Ar-C-H), 121.43 (Ar-C-H), 125.93 (Ar-C-H), 126.05 (Ar-C-H), 126.14 (Ar-C-H), 126.20 (Ar-C-H), 127.43 (Ar-C-H), 127.48 (Ar-C-H), 128.18 (Ar-C-H), 128.35 (quaternary C), 128.60 (Ar-C-H), 128.82 (Ar-C-H), 136.53 (quaternary C), 137.39 (quaternary C), 138.02 (quaternary $\mathbf{C}$ ), 138.15 (quaternary C), 169.27 (CO), 169.71 (CO); $m / z 369.19$ (43.6\%, $\mathrm{M}^{+}$), 321.23 (11.4), 273.13 (77.2), 169.08 (90.9), 168.08 (56.6), 121.06 (79.9), 91.04 (81.4), 77.04 (37.6).

\section{1-Benzyl-8-methyl-9-methylene-2-oxo-2,3,8,8a,9,10,11,11a-octahydro-1H-pyrrolo[2,3-d]}

carbazole-8a-carbonitrile (7). Colourless oil ( $0.14 \mathrm{~g}, 16 \%)$; (Found $\mathrm{M}^{+}, 369.18666, \mathrm{C}_{24} \mathrm{H}_{23} \mathrm{~N}_{3} \mathrm{O}$ requires 369.18411); $v_{\max } / \mathrm{cm}^{-1} 2924.4,2854.8(\mathrm{Ar}-\mathrm{H}), 2218.2(\mathrm{CN}), 1694.1\left(\mathrm{CONR}_{2}\right) ; \delta_{\mathrm{H}}(300$ $\left.\mathrm{MHz} ; \mathrm{CD}_{3} \mathrm{OD}\right) 1.32\left(1 \mathrm{H}, \mathrm{m}, \mathrm{NCHCH}_{2}\right), 2.16-2.28\left(2 \mathrm{H}, \mathrm{m}, \mathrm{NCHCH}_{2} \& \mathrm{NCHCH}_{2} \mathrm{CH}_{2}\right), 2.39$ $\left(1 \mathrm{H}, \mathrm{m}, \mathrm{NCHCH}_{2} \mathrm{CH}_{2}\right), 2.63\left(3 \mathrm{H}, \mathrm{s}, \mathrm{NCH}_{3}\right), 2.69 \& 2.93\left(2 \mathrm{H}, \mathrm{ABq}, \mathrm{CH}_{2} \mathrm{CO}\right), 3.13(1 \mathrm{H}, \mathrm{dd}, J$ 5.4, NCH), $4.11 \& 4.76\left(2 \mathrm{H}, \mathrm{ABq}, J 14.7, \mathrm{CONCH}_{2} \mathrm{Ar}\right), 5.49\left(1 \mathrm{H}, \mathrm{d}, J 1.3, \mathrm{CCH}_{2}\right), 5.63(1 \mathrm{H}, \mathrm{s}$, $\left.\mathrm{CCH}_{2}\right), 6.67(1 \mathrm{H}, \mathrm{d}, J 7.5, \mathrm{C}-7 H), 6.76(1 \mathrm{H}, \mathrm{td}, J 7.5 \& 1.0, \mathrm{C}-6 H), 6.94(1 \mathrm{H}, \mathrm{d}, J 7.4, \mathrm{C}-4 H)$, $7.16(1 \mathrm{H}, \mathrm{td}, J 7.5 \& 1.0, \mathrm{C}-5 H), 7.20(5 \mathrm{H}, \mathrm{m}, \mathrm{Ar}-H) ; \delta_{\mathrm{C}}\left(75 \mathrm{MHz} ; \mathrm{CD}_{3} \mathrm{OD}\right) 25.53\left(\mathrm{CH}_{2}\right), 29.38$ $\left(\mathbf{C H}_{2}\right), 30.69\left(\mathrm{NCH}_{3}\right), 38.38\left(\mathbf{C H}_{2}\right), 45.77\left(\mathbf{C H}_{2}\right), 53.84$ (quaternary $\left.\mathbf{C}\right), 65.02(\mathrm{NCH}), 78.91$ (quaternary C), 110.57 (C-7), $116.90(\mathbf{C N}), 119.43\left(\mathrm{CCH}_{2}\right), 121.58$ (Ar-C-H), 122.59 (Ar-C-H), 128.87 (Ar-C-H), 129.62 (2 x Ar-C-H), 129.69 (2 x Ar-C-H), 130.55 (Ar-C-H), 134.66 (quaternary C), 137.19 (quaternary C), 140.86 (quaternary C), 149.83 (quaternary C), 173.36 (CO); $m / z 369.19$ (100.0\%, M ${ }^{+}$), 360.95 (16.4), 266.99 (24.4), 169.08 (35.8), 149.02 (32.9), 91.06 (86.6), 83.09 (35.2).

\section{2-Benzyl-3-methylene-11-oxo-1,2,3,3a,4,5,10,11,12,12a-decahydropyrrolo[2,3-n]}

phenanthridine-3a-carbonitrile (13). White solid (0.13 g, 15\%); m.p. 195-197 ${ }^{\circ} \mathrm{C}$; (Found $\mathrm{M}^{+}$, $369.18407, \mathrm{C}_{24} \mathrm{H}_{23} \mathrm{~N}_{3} \mathrm{O}$ requires 369.18411$) ; \delta_{\mathrm{H}}\left(300 \mathrm{MHz} ; \mathrm{CDCl}_{3}\right) 1.71-1.62\left(1 \mathrm{H}, \mathrm{m}, \mathrm{NCHCH}_{2}\right)$, 1.97-1.85 (1H, m, $\left.\mathrm{NCHCH}_{2}\right), 2.35\left(2 \mathrm{H}, \mathrm{m}, \mathrm{NCHCH}_{2} \mathrm{CH}_{2}\right), 2.84 \& 2.98(2 \mathrm{H}, \mathrm{ABq}, J 17.5$, $\left.\mathrm{CH}_{2} \mathrm{CO}\right), 3.37\left(1 \mathrm{H}, \mathrm{dd}, J 12.6 \& 3.3, \mathrm{NCH}_{2}\right), 3.52\left(1 \mathrm{H}, \mathrm{d}, J 12.6, \mathrm{NCH}_{2}\right), 3.54(1 \mathrm{H}, \mathrm{t}, J$ 5.3, $\mathrm{NCH}), 4.04\left(1 \mathrm{H}, \mathrm{d}, J 14.9, \mathrm{CONCH}_{2} \mathrm{Ar}\right), 4.19(1 \mathrm{H}$, br s, $\mathrm{NH}), 4.96\left(1 \mathrm{H}, \mathrm{d}, J 14.9, \mathrm{CONCH}_{2} \mathrm{Ar}\right)$, $5.31\left(1 \mathrm{H}, \mathrm{s}, \mathrm{CCH}_{2}\right), 5.62\left(1 \mathrm{H}, \mathrm{s}, \mathrm{CCH}_{2}\right), 6.54(1 \mathrm{H}, \mathrm{d}, J 7.5, \mathrm{C}-7 \mathrm{H}), 6.69(1 \mathrm{H}, \mathrm{td}, J 7.5 \& 1.0, \mathrm{C}-$ 
5H), 7.01-7.07 (2H, m, Ar- $H), 7.13-7.24(5 \mathrm{H}, \mathrm{m}, \mathrm{Ar}-H) ; \delta_{\mathrm{C}}\left(75 \mathrm{MHz} ; \mathrm{CDCl}_{3}\right) 24.57\left(\mathrm{CH}_{2}\right), 25.38$ $\left(\mathbf{C H}_{2}\right), 42.91\left(\mathbf{C H}_{2}\right), 43.01$ (quaternary $\left.\mathbf{C}\right), 44.70\left(\mathbf{C H}_{2}\right), 45.65$ (quaternary $\left.\mathbf{C}\right), 48.91\left(\mathbf{C H}_{2}\right)$, $62.13(\mathrm{NCH}), 115.54(\mathbf{C}-7), 116.99\left(\mathrm{CCH}_{2}\right), 119.20(\mathrm{Ar}-\mathrm{C}-\mathrm{H}), 120.12$ (quaternary $\left.\mathbf{C}\right), 125.53$ (quaternary C), 126.58 (Ar-C-H), 127.75 (Ar-C-H), 128.35 (2 x Ar-C-H), 128.55 (Ar-C-H), 128.62 ( 2 x Ar-C-H), 135.67 (quaternary C), 140.27 (quaternary C), 140.87 (quaternary C), $172.60(\mathrm{CO}) ; \mathrm{m} / \mathrm{z} 369.18\left(48.0 \%, \mathrm{M}^{+}\right), 303.15$ (21.7), 266.99 (27.8), 245.11 (29.2), 169.08 (53.2), $91.06(28.8)$.

\section{Acknowledgements}

We wish to acknowledge Kingston University for the financial support to carry out this research.

\section{References and Footnotes}

1. Bonjoch, J.; Sole, D. Chem. Rev. 2000, 100, 3455.

2. McCarthy, C.; Jones, K. J. Chem. Soc., Chem. Commun. 1989, 1717.

3. Hilton, S. T.; Ho, T. C. T.; Pljevaljcic, G.; Schulte, M.; Jones, K. J. Chem. Soc., Chem. Commun. 2001, 209.

4. Fukuyama, T.; Jow, C.; Cheung, M. Tetrahedron Lett. 1995, 36, 6373.

5. Bowman, W. R.; Coghlan, D. C. Tetrahedron 1997, 53, 15787.

6. Hilton, S. T.; Ho, T. C. T.; Pljevaljcic, G.; Jones, K. Org. Lett. 2000, 2, 2639.

7. Curran, D. P.; Shen, W. J. Am. Chem. Soc. 1993, 115, 6051.

8. Lathbury, D. C.; Parsons, P. J.; Pinto, I. J. Chem. Soc., Chem. Commun. 1988, 81.

9. Curran, D. P.; Kim, D.;Liu, H. T.;Shen, W. J. Am. Chem. Soc. 1988, 110, 5900.

10. Abeywickrema, A. N.; Beckwith, A. L. J.; Gerba, S. J. Org. Chem. 1987, 52, 4072. Bowmann, W. R.; Westlake, P. J. Tetrahedron 1992, 48, 4027. Jones, K.; Brunton, S. A.; Gosain, R. Tetrahedron Lett. 1999, 40, 8935.

11. Intramolecular addition of an alkyl radical to an aromatic ring has been shown to occur, see for example Beckwith, A. L. J.; Storey, J. M. D. J. Chem. Soc., Chem. Commun. 1995, 977. 